\title{
Modeling the impact of supra-structural network nodes: The case of anonymous syringe sharing and HIV among people who inject drugs
}

\section{Lindsey Richardson, ${ }^{\text {a,1 }}$ Nuffield College, University of Oxford Thomas Grund, ${ }^{b, 2}$ Nuffield College, University of Oxford}

${ }^{a}$ New Road, Oxford, UK OX1 1NF, lindsey.a.richardson@gmail.com

b New Road, Oxford, UK OX1 1NF, thomas.u.grund@gmail.com

\author{
Corresponding Author: $\quad$ Lindsey Richardson \\ Urban Health Research Initiative \\ British Columbia Centre for Excellence in HIV/AIDS \\ St. Paul's Hospital \\ 608 - 1081 Burrard Street \\ Vancouver, BC, Canada \\ +1 (604) 418-5321 \\ lindsey.a.richardson@gmail.com
}

Tables: 5

Figures: 2

Running Head: Shooting Galleries and HIV transmission

\footnotetext{
${ }^{1}$ Present Address: Urban Health Research Initiative, British Columbia Centre for Excellence in HIV/AIDS, St. Paul's Hospital, 608-1081 Burrard Street, Vancouver, BC, Canada, V6Z 1 Y6

${ }^{2}$ Present Address: ETH Zurich, Chair of Sociology in particular Modeling and Simulation, Clausiusstrasse 52, 8092

Zurich, Switzerland
} 


\begin{abstract}
Networks are well understood as crucial to the diffusion of HIV among injection drug users (IDU), but quasi-anonymous risk nodes - such as shooting galleries - resist measurement and incorporation into empirical analyses of disease diffusion. Drawing on network data from 767 IDU in Bushwick, Brooklyn, we illustrate the use of calibrated agent-based models (CABM) to account for network structure, injection practices, and quasi-anonymous transmission in shooting galleries. Results confirm the importance of network structure and actor heterogeneity to the magnitude and speed of HIV transmission. Models further demonstrate that quasi-anonymous injections in shooting galleries increase the speed of HIV diffusion and have the greatest impact on HIV seroconversion levels for IDU at the network periphery. Shooting galleries are shown to be transmission hubs that operate independently of traceable structural ties, linking otherwise unconnected network components. CABMs demonstrate potentially increased understandings of HIV diffusion dynamics by infusing computer simulations with empirical data.
\end{abstract}

Keywords: Calibrated agent based model, disease diffusion, HIV, quasi-anonymous transmission, shooting gallery, syringe sharing 


\section{Introduction}

The injection of illicit drugs is associated with various harms, including the spread of blood-borne disease such as HIV (Aceijas et al., 2004). While HIV infection among injection drug users (IDU) has decreased in some settings, such as the US (Hall et al., 2008), in other contexts, including Eastern Europe and Central Asia, rates of transmission among IDU remain alarmingly high (UNAIDS, 2010). Networks are crucial to understanding the diffusion of HIV infection among IDU. The mixing patterns of individuals, when combined with the non-random distribution of HIV-risk behaviors within social networks, have demonstrated that the structure of social networks has implications for HIV transmission over and above the effects of individual characteristics and behaviors (Klovdahl, 1985; Friedman, 1995; Potterat et al., 1999).

Despite the importance of network structures to disease diffusion, less is known about the role and impact of quasi-anonymous disease transmission hubs such as shooting galleries, where individuals know little or nothing about their injection partners (Friedman et al., 2007). Shooting galleries are often omitted from models depicting the spread of HIV disease. They transcend the traceable structures of the network and form supra-structural nodes having potentially significant impacts on patterns of disease diffusion. Existing studies that consider these dedicated mixing sites generally use compartmental or differential equation (DE) models (see, for example, Volz et al., 2011). We propose the use of another promising methodological strategy - calibrated agent based modeling $(\mathrm{CABM})$ - to isolate the potential effect of shooting galleries on HIV diffusion in risk networks of IDU. The purpose of the current study is therefore to apply calibrated agent based models, a network-based analytical strategy, to explore how suprastructural network nodes impact the transmission of HIV in a network of IDU. 
Previous research has established the role of social relationships in the diffusion of HIV among IDU, demonstrating that they matter for both individual risk behaviors (Tyler, 2008) and HIV infection (Goodreau, 2006). Social influence has been suggested as a crucial mechanism by which network structure and levels of social integration predict individual health outcomes (Berkman et al., 2000). This influence may operate, for example, through processes related to the diffusion of individual behavior or norms (Rogers, 2003; Latkin et al., 2010) or personal network exposure, which describes the extent to which a network member is exposed to a particular practice (Valente, 2005; Gyarmanthy and Neiagus, 2005). Empirically, drug using practices of "alters" (i.e. an individual with whom a person shares a tie, here an injecting partner), consistently predict the drug using practices of network "egos" (i.e. the individual in question) in terms of syringe sharing, shooting gallery attendance, renting syringes and sharing other equipment, such as drug cookers (Neaigus et al., 1994; Latkin et al., 1995).

Other research illustrates that the structure of drug user networks is important for disease diffusion. Existing studies suggest that the spread of HIV infection is contingent upon network structure, behavior associated with increased HIV risk, and the location of HIV infiltration and concentration within the network (Curtis et al., 1995; Price et al., 1995; Rothenberg et al., 1995; Rothenberg et al., 2001). For example, in Colorado Springs, HIV positive members of the IDU network are peripherally and weakly connected to larger network chains, suggesting a network structure that does not facilitates active propagation (Rothenberg et al., 1995). In St. Louis, HIV is concentrated among injectors whose close social connections are exclusively with other drug injectors. In this case, isolated, closed networks may reduce viral spread (Price et al. 1995). IDU in Atlanta connect heterosexual and men-who-have-sex-with-men (MSM) populations, thereby indirectly driving extensive HIV transmission (Volz et al., 2010). In New York research 
identifies large, densely connected network components that include a network core with proportionately higher HIV prevalence rates than other network members (Curtis et al., 1995), thereby facilitating greater and more rapid spread of HIV throughout the network. Spread may also depend on the location and connectivity of individuals with relatively more infectious acute HIV infections (Friedman et al., 2000).

Despite the identification of highly consequential network characteristics, networkfocussed studies of HIV diffusion among IDU face considerable obstacles, including difficulties obtaining reliable and complete network data for IDU. Linkages between network members may be short term and between people who either do not have reliable data about one another or who are reticent to name their associates. IDU may avoid identification by researchers or other officials because of the illegal nature of some of their activities. Subjects may also have difficulty recalling with whom they have interacted and of what their interactions were comprised (Friedman, 1995). Further, variability in the duration and content of linkages between network members makes it difficult and highly resource intensive to account for network dynamics over time. The dynamic, concealed and potentially chaotic nature of IDU networks makes them hard to reliably quantify.

Particularly relevant to the current study are injection environments that present particular challenges for the mapping and modeling of network processes, such as shooting galleries. Shooting galleries are clandestine locations where the rental and multi-person use of injection equipment commonly occurs. Shooting galleries form and dissolve rapidly and unpredictably, and may involve IDU that are unknown to each other and otherwise unconnected in the broader risk network. Shooting galleries have been identified as HIV 'risk environments' (Rhodes et al., 2005) and have been associated with non-recreational, high intensity drug 
injection and higher exposure to HIV disease and substantially elevated risk for HIV infection (Chitwood et al., 1990; Des Jarlais and Friedman, 1990; Klein and Levy, 2003). HIV transmission in shooting galleries may occur through the sharing of injection equipment between shooting gallery attendees who may or may not otherwise know each other or through the use or rental of previously used injection equipment. Previous research acknowledges the relevance of such "quasi-anonymous risk nodes" (Friedman et al., 2007: 645), which include group sex events, bathhouses and shooting galleries. Similarly, other studies have identified supra-individual network components that are important to disease diffusion, notably Klovdahl et al.'s (2001) seminal study of tuberculosis transmission in public places. In these contexts, conventional probes to name network members fail to identify entire sections of an individual's risk network (Friedman, 1995). Quasi-anonymous risk nodes therefore resist measurement and incorporation into empirical network analyses of disease diffusion.

These challenges often prompt researchers to use simulation models of HIV transmission among IDU (Allard, 1990, Peterson et al., 1990; Iannelli et al., 1997). Many of these studies take contact and/or network structure into account. Kretszschmar and Wiessing (1998) demonstrate how random versus non-random mixing greatly elevates HIV prevalence. Zaric (2002) also compares the impact of random vs. non-random mixing, noting that the assumption of random mixing may lead to higher incidence and prevalence but is generally not an appropriate modeling assumption. Kiss et al. (2008) compare assortative vs. disassortative mixing patterns on epidemic dynamics, demonstrating that with assortative mixing, outbreaks occur at lower infection rates with faster epidemic growth and shorter epidemic duration. Other simulations take shooting galleries or dedicated mixing sites into account. For example, Kaplan (1989) models a homogeneously mixing population in which all needles are shared in randomly selected shooting 
galleries. Greenhalgh and Hay (1997) incorporate the effect of behavior change on transmission, showing a reduction in HIV transmission when injection equipment is cleaned after use. Atkinson (1996) simulates HIV spread in a single high-volume shooting gallery with varying numbers of partners and injection frequencies. These studies generally use compartmental or differential equation (DE) models that aggregate individuals into compartments within which they are considered to be homogeneous and well mixed (Zaric 2002; Rahmandad and Sterman, 2008). However, a systematic comparison of agent based and DE models in the context of diffusion suggests that ABMs are better able to examine heterogeneity in individual attributes and network structures (Rahmandad and Sterman, 2008). They are also better equipped to represent complex social structure or mixing patterns that depend on network structure (Zaric, 2002), and have been recently employed to effectively describe drug markets and HIV transmission patterns as complex adaptive systems (Hoffer et al., 2009; Cui et al., 2009).

To our knowledge no one has to date quantified how shooting galleries impact transmission dynamics by demonstrating the impact of shooting galleries as quasi-anonymous risk nodes in a network context. This is a potentially consequential omission: shooting galleries combine multiple epidemiologically important network characteristics including concurrent injection partnerships (Watts and May, 1992; Morris and Kretzschmar, 1997; Curtis et al., 1995) involving core network members (Morris, 1995; Friedman et al., 1997a; Goodreau, 2006) who act as bridges (Morris et al., 1996; Volz et al., 2010) across otherwise unconnected parts of the risk network. The current study outlines a calibrated agent based modeling strategy that combines both network analysis and information about individual injection behavior to characterize the role of shooting galleries as quasi-anonymous risk nodes that impact HIV diffusion patterns and incidence levels among IDU. 


\section{Material and Methods}

Agent-based modeling (ABM) simulates the interactions of individual agents who are connected to each other in social, structural or normative environments whereby individual behaviors, decisions and interactions at the micro-level are shown to produce broader macrolevel trends (Gilbert, 2008). They are distinct from other types of simulations by generating outcomes and predictions from bottom-up, that is, as product of individual 'simulated' behavior (Gilbert, 2008). Only a limited number of ABMs have addressed HIV infection (Alam et al., 2007; Cui et al., 2009).

While ABMs have traditionally been employed in theoretical contexts (Macy and Willer, 2002), data driven ABMs are becoming increasingly common, and the ability of ABMs to incorporate empirical data warrants further attention (see Cioffi-Revilla, 2010, Hedström, 2006, Janssen and Ostrom, 2006, Moss, 2008, Poteete et al., 2010, Windrum et al., 2007). In so-called calibrated agent-based models (CABMs), real-world information is used to inform model assumptions. Because different, often arbitrary, model specifications can significantly alter the interplay of agents and their environments, ungrounded model specifications can inappropriately drive results. Emulating a real-world context addresses such difficulties by empirically justifying model design and decreasing the number of assumed parameters. Model calibration also allows for richer model specifications, particularly those related to actor and network heterogeneity. In addition, in situations where complete socio-demographic and network information about individuals is difficult to obtain, but some overall population information is available, as in the case of IDU, CABM's permit variation in the dimensional complexity of a model while holding general population aggregates at constant values. By doing so, the relevance of certain micro- 
structures and micro-mechanisms for the observed outcome can be disentangled. This is the strategy applied in the current study.

\subsection{Real-world context}

We derive a set of nested models that have the same population values for network size, number of injections, number of injections with shared syringes and overall number of injecting partners or links in the network. These values are derived from existing studies and are kept constant at the aggregate level in all models. As we increase model complexity, we redistribute these quantities according to subgroup-specific information that is likewise empirically substantiated. Incremental recalibration for each successive model isolates the effects of a particular change on the rate and magnitude of total and proportional HIV diffusion. Models remain comparable as a result.

Data used to derive these overall population values come predominantly from published studies of the 'Social Factors and HIV Risk' (SFHR) study conducted in Bushwick, Brooklyn (Curtis et al., 1995, Friedman et al., 1997a, Friedman et al., 1999, Kottiri et al., 2002). The study employed targeted sampling and chain referral between July 1991 and January 1993 to recruit people who had injected drugs in the prior 12 months. Data from the study include information on drug use, HIV risk behavior, demographic characteristics, network structure and roles in the street economy. Curtis et al. (1995), Neaigus et al. (1995) and Friedman et al. (1999) provide detailed descriptions of this data and information about three subgroups within the population: a core group, an inner periphery and an outer periphery. They also provide estimates about the average number of injecting partners (i.e. potential syringe sharers) in each of these three groups during the last 30 days. Core members are high intensity drug users who are more likely to 
engage in high risk behavior with both seropositive and seronegative injectors, share drugs with each other, be distributors, use shooting galleries, and sell syringes to other people. Inner periphery members inject less frequently, but are generally daily injectors who often share drugs, attend shooting galleries and share syringes at levels marginally lower than that of core network members. Outer periphery members are much less likely to use shooting galleries and share syringes, report lower levels of injection and injection partnerships, and often purchase drugs in the neighborhood that they then use in their own homes. Information about individual injection and injection-related risk behavior is reported for the last 30 days, and in order to optimize calibration efforts, simulations are run using this monthly time scale. This strategy avoids additional manipulation of existing data such as, for example, the non-trivial determination of the number of individuals' injection partners in a single day, or the recurrence of syringe sharing between partners across a smaller time scale. Implications of this modeling decision are further discussed in the limitations section.

The Bushwick studies do not provide estimates of how often IDU who report syringe sharing or shooting gallery attendance actually participate in these practices, it being unlikely that IDU who report these behaviors do so for all injections. We therefore rely on other studies of similar populations to derive estimates of the proportion of total injections that involve the sharing of syringes to be between $30 \%$ and 50\% (Laufer, 2001; Wood et al., 2001; Ouellet et al., 2004). Based on these estimates the current analysis constructs a group-specific gradient (core $=$ $50 \%$, inner periphery $=40 \%$, outer periphery $=30 \%$ ). Shooting gallery usage is derived similarly. For those that do not share injecting equipment and those who do not attend shooting galleries, the probabilities of these activities are set to zero. 
Tables 1 and 2 give an overview of the empirically substantiated scores that we use for model calibration and the studies from which these scores were retrieved. We also use this information to derive overall total population values for the parameters at hand (such as the total number of injections in the network). Population aggregate values are summarized in Table 3 and include the total population size (767), the number of syringe sharers (163), the number of shooting gallery users (146), the total number of injections in one month (72055) and the total number of non-directed links in the network (661).

\subsection{Analytical strategy and models}

Our strategy is to develop a series of four CABMs that explore how diffusion patterns change depending on the level of structural complexity accounted for in the model. Keeping the aggregate population values constant in all models, we incrementally redistribute aggregate information across the core, inner periphery and outer periphery according to the subgroupspecific information described above. We subsequently examine the effect of these redistributions. Because the overall socio-metric information is the same, models are comparable and the differences in outcomes between models are uniquely attributable to differences in network structure and actor heterogeneity. The first three models describe HIV transmission accounting for network and actor heterogeneity. The fourth model incorporates shooting galleries as quasi-anonymous risk nodes.

\subsubsection{Transmission of HIV through the network}

In the first three models, the probability for HIV seroconversion of an agent $i$ at time $t$ is determined as follows: $T$ equals the probability of infection given injection with a shared, 
contaminated syringe, $0 \leq T \leq 1 . C C_{i t}$ is the number of critical contacts, or number of times $i$ could have been infected at time $t$.

$$
\begin{aligned}
& \operatorname{Pr}\left(\mathrm{HIV}_{\mathrm{it}}=0 \mid \mathrm{HIV}_{\mathrm{it}-1}=0\right)=1-\mathrm{Pr}\left(\mathrm{H} \mathrm{V}_{\mathrm{it}}=0 \mid \mathrm{H}\left[\mathrm{V}_{\mathrm{i}-1}=0\right) ;\right. \\
& \operatorname{Pr}\left(\mathrm{HIV}_{\mathrm{it}}=0 \mid \mathrm{HIV}_{\mathrm{it}-1}=0\right)=(1-\mathrm{T})^{\mathrm{Cc}} \mathrm{st}
\end{aligned}
$$

The critical contacts $C C_{i t}$ are limited by $i$ 's injection frequency and how risky these injections are. We define

$$
\mathrm{CC}_{\mathrm{tt}}=\mathrm{I}_{1} \cdot \mathrm{R}
$$

where $I_{i}$ is the number of injections of agent $i$ during time period $t$ and $R_{i t}$, or the risk factor, is a number that ranges from zero to one which is determined by (a) how often agent $i$ shares syringes, and (b) how many of $i$ 's injection partners are HIV seropositive. $R_{i t}$ reaches its maximum (=1) when all injection partners of $i$ are HIV seropositive at time $t-1$ and when $i$ shares syringes all the time. When none of $i$ 's injecting partners have HIV, or agent $i$ never shares syringes, this factors is zero. It is formally defined as:

$$
\mathrm{R}_{\mathrm{tt}}=\mathrm{SH}_{\mathrm{l}} \cdot \sum_{\mathrm{j}=1}^{\mathrm{v}}\left(\mathrm{X}_{\mathrm{I}} \cdot \mathrm{HIV}_{\mathrm{tt}-1} \cdot \frac{\mathrm{l}_{\mathrm{j}}}{\sum_{\mathrm{l}=1}^{\mathrm{X}=1}\left(\mathrm{X}_{\mathrm{ix}} \cdot \mathrm{l}_{\mathrm{k}}\right)}\right) ;
$$

where $S H_{i}$ is the proportion of injections where agent $i$ shares syringes, $0 \leq S H_{i} \leq 1$; $N$ is the total number of agents; $X_{i j}$ indicates whether $i$ and $j$ are injecting partners ( 1 if they are linked, 0 otherwise; in the model network links are undirected and assumed to involve both receptive and distributive syringe sharing) and $H I V_{j t-1}$ indicates the HIV status of agent $j$ at time $t-l$ ( 1 when $j$ is HIV positive in $t-1,0$ otherwise). The risk factor represents the share of overall injections with network links $j$ that could potentially cause HIV infection. The risk factor is therefore determined by the probability of $i$ sharing syringes with $j$ at time $t$, the HIV status of agent $j$ at time $t-1$, and the relative importance of agent $j$ in the ego-network of $i$. The last component reflects the idea 
that the probability that agent $i$ will inject with $j$ is higher when $j$ is a heavy-user. Here, we assume that the relative importance of $j$ for $i$ is proportional to the injection frequency of $j$ relative to the injection frequency of all other acquaintances of $i$.

The first model is the most basic, with no differentiation occurring between agents or network components. The population has 767 agents, with 661 non-directed links between them. The network is constructed under a mixing assumption where links are formed randomly. Agent attributes are likewise randomly assigned across all individuals. A link between two agents represents a relationship in which the two have injected drugs together in the prior 30 days, but may or may not share syringes. The total number of injections (72055) is equally distributed amongst the 767 agents resulting in 93.94 injections per person per month. The total number of syringe sharers is set to 163 . In order to keep the actual injections with shared syringes constant at 7580 , the probability for each of the 163 syringe sharers to share syringes at any given injection is calculated as $\frac{7580}{163 \cdot 93.94} \approx 0.49$.

The second model incorporates additional information about the average number of links (see Table 1) and the mixing preferences (see Table 2) for agents in each of the core, inner periphery and outer periphery sub-groups. Core members have a mean number of 12.8 links, inner periphery an average of 5.2 links and outer periphery members 0.5 links on average (Curtis et al., 1995). The algorithm used to draw the network from this information is based on the following steps: (1) each agent is assigned a preferred number of links (or link quota) depending on their group membership, normally distributed around the mean for each group with a standard deviation of 0.1 multiplied by the mean; (2) a random agent (ego) is chosen and the preferred group for a link for that IDU is identified, based on the description of the network in Curtis et al. (1995) (see Table 3); (3) a member of the ego's preferred group who has also not yet filled their 
link quota is selected (alter); if no member of the preferred group is available then another random agent that has not filled its quota is randomly selected and (4) a link is established between ego and alter, which increases the number of realized links for both ego and alter. As in Model 1, monthly injections are distributed at random over the population and there is no difference from Model 1 in terms of syringe sharing and shooting gallery usage. Model 2, when compared to Model 1, isolates the effect of non-random network structure where the number of links per person depends on the membership of individuals in the core, inner periphery or outer periphery sub-groups.

\section{TABLE 1 AROUND HERE}

\section{TABLE 2 AROUND HERE}

In the third model, we additionally calibrate the injection behavior of agents according to their group membership using the data described in Table 1. Instead of all agents having the same number of injections per month, core group members inject an average of 395 times, inner periphery an average of 193 times and outer periphery group members inject an average of 60 times per month. Information from the Bushwick studies is also used to redistribute the 163 individuals who share syringes into the three subgroups, with 15 core, 33 inner periphery and 115 outer periphery syringe sharing IDU. The probabilities that a syringe sharing IDU actually shares a syringe for a given injection are set to the $0.5,0.4$ and 0.3 across the core, inner periphery and outer periphery respectively. This results in the same 7580 total network injections with shared syringes in every 30-day cycle. Individual behavior is modelled with a random component, drawing the number of injections per individual and the proportion of shared syringes among sharers from normal distributions around the sub-group means. Comparing Model 3 to Model 2 isolates the effect of group specific injection behavior on disease diffusion. 


\subsubsection{HIV transmission in shooting galleries}

The final model, which incorporates shooting gallery participation, adds the additional consideration of the proportion of a shooting gallery users' injections that actually occur in the shooting gallery for both agent $i$ and all agents $k$ with whom agent $i$ injects. Here the number of critical contacts of an agent $i$ at time $t$ depends not only on the HIV infections in the ego-network of $i$, but also on that of anonymous contacts. It is given by:

$$
\mathrm{CC}_{\mathrm{tt}}=\left(1-\mathrm{SG}_{1}\right) \cdot \mathrm{I}_{1} \cdot \mathrm{R}_{\mathrm{tt}}+\mathrm{SG}_{1} \cdot \mathrm{I}_{1} \cdot \mathrm{RG}_{\mathrm{i}} ;
$$

where $S G_{i}$ represents the proportion of injections for agent $i$ that occur in shooting galleries, $I_{i}$ represents the total monthly injections of $i, R_{i t}$ is the risk factor of agent $i$ at time $t$ and $R G_{i t}$ is a risk factor specific to shooting gallery injections. This is defined as:

$$
\mathrm{RG}_{\mathrm{I}:}=\mathrm{SH}_{1} \cdot \sum_{\mathrm{l}=1}^{\mathrm{N}}\left(\frac{\mathrm{L} \cdot \mathrm{SG}_{1}}{\sum_{\mathrm{k}=1}^{\mathrm{N}}\left(\mathrm{I}_{\mathrm{k}} \cdot \mathrm{SG}_{\mathrm{k}}\right)} \cdot \mathrm{H}\left[\mathrm{V}_{\mathrm{jt}-\mathrm{z}}\right)\right.
$$

where $S H_{i}$ is the proportion of injections where $i$ shares syringes, $S G_{i}$ is the proportion of $i$ 's injections that take place in a shooting gallery, $I_{i}$ is the number of monthly injections of $i$ and $H I V_{j t-1}$ is the HIV status of $j$ at time $t-1$. Again, the idea is to weight the importance of others by their injection frequency. In this case this is the number of times that they inject in a shooting gallery. The denominator of the first bracketed term represents the total number of injections of all agents $k$ that take place in a shooting gallery. When the numerator of this term, representing the total number of injections of a single agent $j$ taking place in shooting galleries, is divided by the denominator, we get the relative importance of actor $j$ in the shooting gallery scene. Taken cumulatively across the whole network, we thereby account for the fact that some people are more likely to be met in shooting galleries than others. 
We know that 20 core group, 34 inner periphery and 92 outer periphery group members report injecting in a shooting gallery. Again, we draw on reasonable assumptions (see Table 1) to model a gradient in terms of actual probabilities of a given injection taking place in a shooting gallery. We assume these probabilities to be 0.5 (core), 0.4 (inner periphery) and 0.3 (outer periphery) for those who attend shooting galleries and zero for those who do not. A total of 8231 injections take place in shooting galleries, though not all are with shared syringes. Model 4 also incorporates individual level variation around the subgroup means. Contrasting Model 4 with Model 3 allows us to isolate the effect that quasi-anonymous shooting galleries have on disease diffusion patterns. Table 4 summarizes the specification of all four models.

\subsection{Additional considerations}

In disease diffusion simulations, it is generally acknowledged that there is a trade-off between maintaining simplicity in order to allow the process and effects to be clearly understood, and representing the complexity inherent in the social environment (Sallach and Macal, 2001, Raboud et al., 2003; Goodreau, 2006). In order to examine the independent effects of anonymous syringe sharing in the absence of confounding factors, we intentionally omit a number of factors, assuming no movement in and out of the population because of death or migration (Zaric, 2002; Raboud et al., 2003), no network change in time, and socio-demographically indistinguishable actors, even though there is some evidence of differences in syringe sharing practices and shooting gallery attendance along demographic and socio-economic lines (Sherman et al., 2001; Pouget et al., 2005). Like Kretzschmar and Wiessing (1998) we set the rate of infectivity per shared syringe at 0.02 among serodiscordant injection partners, assuming, as in prior studies, no 
variable infectivity (Morris and Kretzschmar, 1997, Goodreau, 2006) despite the considerable evidence that infectivity varies according to the stage of infection (Longini Jr et al., 1990). These assumptions make it possible to state that differences in outcome are due solely to the parameters examined in each successive model. The unit of time $t$ in our study is set at one month to correspond with the empirical information available for calibration. While monthly time intervals are reasonable periods for the modeling of injection behavior, within-month dynamics are ignored by this assumption.

In all models we infect six seeds with HIV and observe disease diffusion patterns. In Model 2 to 4, these seeds are equally distributed with two in each of the three sub-groups. Each model specification is simulated for 48 time periods (corresponding to a four year period) and repeated in 1000 simulation runs. The results represent average HIV seroconversion rates across multiple repetitions for a relatively large network, and are thus reliable estimates of the dynamics of HIV transmission over time. Analyses are conducted using NetLogo 4.0 (Wilensky, 1999).

\section{Results and Discussion}

Simulation results are summarized in Table 5, which displays the average seroprevalence levels after 48 months, and shows that as model complexity increases so do levels of HIV seroconversion. In Model 1, where links and injection behavior are randomly and evenly distributed across the network, there is very little diffusion of HIV, with an average of 3.40 individuals who seroconvert in addition to the six HIV seropositive seeds. Since most individuals have only a single link, viral spread is contained by those that do not share syringes. The incorporation of network structure in Model 2 results in an average of 25.55 individuals with HIV after 48 cycles (3\% of the total population). Highly linked, HIV seropositive agents are 
more effective viral transmitters. Despite the total number of HIV positive agents being the smallest in the core group, it has proportionally the highest level of HIV infection of the three network subgroups (17\%). When we calibrate both the network and the injection behavior of agents in Model 3, the magnitude of HIV infection increases dramatically, producing an average of $60.00 \mathrm{HIV}$ seropositive individuals with high proportions amongst both core group members (39\%) and inner periphery group members (30\%). Finally, the incorporation of shooting galleries in Model 4 is also consequential to levels of resultant HIV infections, with an average of 75.54 HIV seropositive individuals at the end of the simulation run. This further increase happens almost uniquely in the outer periphery of the network where the number of infected individuals nearly doubles to 28.15 , while the number of infected core and inner periphery group members increases only slightly.

Figure 1 provides information about the distribution of the number of HIV infections across the 1000 simulation runs conducted for each model. It combines four histograms, one for each of the four models, that indicate how many of the simulation runs produce a given number of HIV infections. The narrow distribution for Model 1 suggests very low levels of HIV diffusion, with generally only two to five HIV seroconversions in the population. The distribution of total HIV infections for Model 2, comparatively, is on average higher and more dispersed, indicating higher variation in results when we account for network structure. Model 3's distribution is narrower than Model 2, indicating less variation in comparatively higher levels of transmission. Finally, the distribution of outcomes for Model 4 is similar to that of Model 3 with a simple shift to the right indicating a greater number of total infections. 
The results of the four CABMs in the current study are depicted through diffusion curves that show the average level and rate of HIV diffusion for each network subgroup across the 48 monthly cycles of each model. Figure 2 displays graphs, two for each model, showing the average number of HIV infections that occur in each subgroup, and the proportion of each subgroup infected across each of the four subgroups. This allows for the comparison of diffusion curves between models by both the absolute number of HIV infections and the rate of infection within each network sub-group. Results for Model 1 suggest that the small numbers of HIV infections that do occur tend to happen in the first few cycles of the simulation. Those for Model 2 indicate slow, steady growth that has not yet stabilized even by the end of the 48-month simulation period, suggesting that the calibrated network structure is capable of sustaining levels of infection over time. Model 3, conversely, suggests rapid initial growth over the first 12-15 months, with levels of infection relatively stable afterwards. Finally, the addition of shooting galleries in Model 4 reduces this initial growth phase of the epidemic to a period of six-eight months, or approximately half of that of Model 3. For the outer periphery of the network in Model 4, HIV infections gradually increase well into the 36-48 month period.

\subsection{Implications of Results}

Networks have long been considered to be important for the diffusion of HIV among IDU. Such network studies, however, underestimate the importance of potentially anonymous disease transmission vectors, like shooting galleries, for disease diffusion. This study proposes a novel methodological strategy - calibrated agent-based modeling - to introduce supra-structural nodes operating as quasi-anonymous transmission hubs within a network paradigm. A series of four 
CABM's demonstrate how HIV diffusion patterns change depending on the level of complexity accounted for in the model. The calibration of computer simulations is particularly appropriate to the exploration of social contexts embedded in networks in which it is possible to obtain only egocentric behavioral information, such as shooting gallery participation. This type of phenomenon, characterized by potential anonymity of HIV transmission and resistance to quantifiable observation, eludes other network oriented research techniques such as name elicitation. In the current analysis, we use CABMs to examine the potential impact that suprastructural nodes have on diffusion patterns, demonstrating not only that shooting galleries impact the rate of transmission of HIV among IDU, but also the ways in which these impacts are felt at different points in time. Here, methodological symbiosis between ethnographic observation and CABMs empirically grounds a systematic exploration of mechanisms responsible for the spread of HIV.

Results demonstrate distinct patterns of HIV diffusion that are contingent on preferential mixing patterns, network location-specific injection behavior and whether or not IDU inject and share syringes in shooting galleries. The findings of the current study are in line with prior studies (Friedman et al., 1999; Zaric, 2002; Goodreau, 2006; Kiss et al., 2008). Notably, the differences in magnitude, speed and distribution of disease transmission found here are only apparent when the impacts of these patterns are assessed as they develop over time. We also demonstrate that disease transmission increases when injection behavior is network location specific, suggesting multiplicative effects when behaviors that are conducive to viral spread systematically map on to network structures known to effectively transmit infectious disease.

Finally, we show not only that shooting galleries impact HIV diffusion, but also how it is that shooting galleries operate as disease transmission hubs once HIV infection is present in a 
given population of IDU. They accelerate the initial rate of transmission among more centrally connected individuals, and sustain a more gradual rate of infection over time among more peripherally connected individuals. Including shooting galleries in the model modifies the structural constraints of the network, creating supra-structural nodes that operate independently of the network's formal ties. HIV transmission is therefore able to bypass conventional structural constraints. As quasi-anonymous risk nodes, shooting galleries are potentially highly effective HIV transmission vectors. They are disproportionally attended by relatively more connected core and inner periphery network members, operate as bridges that connected otherwise distinct network components, and increase the probability of concurrent high-risk injection relationships because of elevated levels of syringe sharing reported to occur within shooting galleries (Chiasson et al., 1987; Des Jarlais et al., 1988). The convergence of multiple network microstructures known to facilitate the transmission of infectious disease in a single injection environment explains why the presence of shooting galleries represents a network condition under which viral spread is efficient, effective and sustained.

The current study involves a number of limitations. Some of these were inherited from the original Bushwick study and two in particular may have resulted in the underestimation of the size of individual risk networks: (1) a social desirability bias from the underreporting of the number of people with whom a participant injected drugs and (2) a research design parameter that limits the number of people an individual can name as a part of their risk network (Kottiri et al., 2002). Other limitations result from intentional omissions of sources of heterogeneity in order to isolate the specific impact of shooting gallery attendance. These include a lack of network entry and exit due to death or migration and a population that is assumed to be sociodemographically homogenous. We also do not account for the sexual transmission of HIV, likely 
resulting in an underestimation of HIV incidence. One omission that warrants further consideration is the use of a constant rate of infectivity over time. While consistent with previous studies, (Kretzschmar and Wiessing, 1998; Morris and Kretzschmar, 1997, Goodreau, 2006), this strategy could result in the underestimation of HIV infection rates, which might plausibly increase given the high intensity injection practices among the network core that, in stages of acute infection, might rapidly increase the transmission of HIV both within the network core and between the network core and more peripheral members of the network. Recent studies have noted that acute infection, where increases in viral load increase individual infectiousness, can occur across early-, mid- and late-stage infection (Abu-Raddad and Longini Jr., 2008). Accurately modeling the impact of acute infection would ideally incorporate detailed serological-level data on individual viral load. Additionally, the networks used in the current model are static for each of the 1000 simulation runs. The implications of this limitation are threefold. First, the accelerated initial transmission rate among core and inner periphery members and more gradual infection rate among outer periphery members could have far-reaching repercussions in larger or interconnected networks. Second, there is no change in group membership or injection norms because of the spread of HIV, ignoring the potential impact of interventions, prevention efforts and changes in injection behavior over time. Third, because there are a limited number of individuals who engage in syringe sharing, the models described are effectively saturation models. This may also be why, compared to an early estimation of transmission rates in New York (Des Jarlais et al., 1989), our models have considerably lower seroprevalence rates. Another limitation is that the 30-day time span for each simulation arguably neglects within-month dynamics, which may impact HIV transmission patterns if there is systematic variation in drug injection, syringe sharing, or shooting gallery attendance within 
this time scale. Finally, we do not differentiate between different shooting galleries, which may vary by injection practices, preferential attendance by particular individuals and the distribution of HIV seropositive agents.

Many of these limitations represent modeling assumptions, which, when relaxed, could provide fertile ground for further research. For example, it is possible to incorporate variation in the infectivity of previously used syringes. The infectivity of a syringe depends on the viral load of the individual that initially uses the syringe, the amount of viable blood in the syringe and the amount of time between the initial use of the syringe by an HIV-infected IDU and the re-use of that syringe by an HIV-uninfected individual, given evidence suggesting that HIV survives in used syringes for a period of approximately 30 days (Abdala et al., 1999). Such heterogeneity in infectivity would most likely emphasize the role of local structures in spreading HIV, and inhibit the spreading of the virus to individuals in the periphery, given these individuals are less likely to interact with individuals in acute stages of infection. Relaxing the assumption that the network is static and closed could also be an interesting avenue for further exploration. Over time there will inevitably be changes in network structure as individual injecting relationships change and as individuals enter or exit the network due to relocation, migration, movement in and out of the justice system, changes in drug use patterns or death. Network dynamics have the potential to both create linkages with other networks outside the system and to change structural constraints within the existing network, and as such could either propagate infection to or from networks outside the system depicted in the current study or confine HIV infection to localized reservoirs, depending on the location, type, and number of changes. Assessing the impacts of these and other model assumptions could prove to be fruitful lines of future inquiry. 


\section{Conclusions}

Shooting galleries, where individuals may share injecting equipment with people they do not know, or inject with used syringes whose previous users are unknown to them, represent high-risk injection environments that may be vectors of quasi-anonymous HIV transmission. The current analysis is able to both isolate the impact of different network dimensions on disease diffusion and to explore the consequences of network structures that simultaneously facilitate concurrency, operate as cores, and create epidemiological bridges. The potential impact on disease diffusion are important: we show that these environments increase both the speed of HIV infection among the most connected in the network and the number of HIV infections among those who are the least well connected.

The use of CABMs to enhance our understanding of phenomena that are difficult to accurately quantify is potentially crucial to a more robust understanding of disease diffusion. The methodological symbiosis demonstrated in the current study allows for the combination of preexisting data with a systematic modeling of the impacts that different dimensions of network structure and network location-specific individual behavior. We are therefore able to directly link how changes in micro-level individual behavior, when combined with structural and suprastructural network components (such as shooting galleries), impact transmission dynamics, resulting in a more detailed understanding of a macro-level phenomenon. Results combine the context-specific acuity of qualitative and ethnographic work with the analytical rigor of quantitative research, and the dynamics of diffusion are shown over time. 


\section{ACKNOWLEDGEMENTS}

We would like to thank the original investigators and participants of the "Social Factors and HIV Risk" study, and Samuel Friedman in particular for comments on an earlier version of the manuscript. We would also like to thank the Pierre Elliott Trudeau Foundation, the Social Sciences and Humanities Research Council of Canada and the Economic and Social Research Council of the United Kingdom for their generous support of our research. 


\section{REFERENCES}

Abdala, N., Stephens, P.C., Griffith, B.P. and Heimer, R., 1999. Survival of HIV-1 in syringes. Journal of Acquired Immune Deficiency Syndromes \& Human Retrovirology, 20, 73-80.

Abu-Raddad L.J., Longini Jr., I.M., 2008. No HIV stage is dominant in driving the HIV epidemic in sub-Saharan Africa. AIDS, 22, 1055-61.

Alam, S.J., Meyer, R., Ziervogel, G., Moss, S., 2007. The impact of HIV/AIDS in the context of socioeconomic stressors: An evidence-driven approach. Journal of Artificial Societies and Social Simulation, 10, 7.

Allard, R., 1990. A mathematical model to describe the risk of infection from sharing injection equipment. Journal of Acquired Immune Deficiency Syndromes, 3, 1010-16.

Atkinson, J., 1996. A simulation model of the dynamics of HIV transmission in intravenous drug users. Computers and Biomedical Research, 29, 338-349.

Aceijas C., Stimson G.V., Hickman M., Rhodes T., 2004. United Nations Reference Group on HIV/AIDS Prevention and Care among IDU in Developing and Transitional Countries, 2004. Global overview of injecting drug use and HIV infection among injecting drug users. AIDS, 18, 2295-2303.

Berkman, L.F., Glass, T., Brissette, I., Seeman, T.E., 2000. From social integration to health: Durkheim in the new millennium. Social Science \& Medicine, 51, 843-857.

Chiasson, R.E., Moss, A.R., Onishi, R., Osmond, D. Carlson, J.R., 1987. Human immunodeficiency virus in heterosexual intravenous drug users in San Francisco. American Journal of Public Health, 77 169-172.

Chitwood, D.D., McCoy, C.B., Inciardi, J.A., McBride, D.C., Comerford, M., Trapido, E., McCoy, V., Page, J.B., Grivvin, J., Fletcher M.A., Ashman, M.A., 1990. HIV 
seropositivity of needles from shooting galleries in south Florida. American Journal of Public Health, 80, 150-152.

Cioffi-Revilla, C., 2010. A Methodology of Complex Social Simulations. Journal of Artificial Social Societies and Social Simulation, 13, 7.

Cui, Q., Wang, J., Tan, J., Li, J. Yang, K., 2009. Exploring HIV/AIDS epidemic complex network of IDU using ABM and GIS. Chinese Control and Decision Conference, 17-19 June 2009, 1090-1095. doi: 10.1109/CCDC.2009.5191828.

Curtis, R., Friedman, S.R., Neaigus, A., Jose, B., Goldstein, M., Ildefonso, G., 1995. StreetLevel drug markets: Network structure and HIV risk. Social Networks, 17, 229-249.

Des Jarlais, D.C., Friedman, S.R., 1990. Shooting galleries and AIDS: Infection probabilities and 'tough' policies. American Journal of Public Health, 80, 142-144.

Des Jarlais, D.C., Friedman, S.R., Stoneburner, R.L., 1988. HIV infection and intravenous drug use: Critical issues in transmission dynamics, infection outcomes, and prevention. Reviews of Infectious Diseases, 10, 151-157.

Friedman, S.R., 1995. Promising social network research results and suggestions for a research agenda. In: R.H. Needle, Coyle, S.L., Genser, S.G., Trotter, R.T. (Eds.), Social Networks, Drug Abuse, and HIV Transmission. NIDA Research Monograph 151, pp. 196-215.

Friedman, S.R., Bolyard, M., Mateu-Gelabert, P., Goltzman, P., Pawlowicz, M.P., Singh, D.Z., Touze, G., Rossi, D., Maslow, C., Sandoval, M., Flom, P.L., 2007. Some data-driven reflections on priorities in AIDS network research. AIDS Behavior, 11, 641-651.

Friedman, S.R., Curtis, R., Neaigus, A., Jose, B., Des Jarlais, D.C., 1999. Social Networks, Drug Injectors’ Lives and HIV/AIDS. New York: Kluwer Academic/Plenum. 
Friedman, S.R., Kottiri, B., Neaigus, A., Curtis, R., Vermund, S.H., Des Jarlais, D.C., 2000.

Network-related mechanisms may help explain long-term HIV-1 seroprevalence levels that remain high but do not approach population-group saturation. American Journal of Epidemiology, 152, 913-922.

Friedman, S.R., Neaigus, A., Jose, B., Curtis, R., Goldstein, M., Ildefonso, G., Rothernberg, R.B., Des Jarlais, D.C., 1997a. Sociometric risk networks and risk for HIV infection. American Journal of Public Health, 87, 1289-1296.

Friedman, S.R., Neaigus, A., Jose, B., Curtis, R., Goldstein, M., Sotheran, J.L., Wenston, J., Latkin, C.A., Des Jarlais, D.C., 1997b. Network and socio-historical approaches to the HIV epidemic among drug injectors. In: Catalan, J., Sherr, L., Hedge, B. (Eds.), The Impact of AIDS: Psychological and Social Aspects of HIV Infection, edited by Jose Catalan, Lorraine Sherr, and Barbara Hedge, Amsterdam, The Netherlands: Harwood Academic, pp. 89-113.

Gilbert, N., 2008. Agent-Based Models. London: Sage.

Goodreau, S.M., 2006. Assessing the effects of human mixing patterns on human immunodeficiency virus - 1: Interhost phylogenetics through social network simulation. Genetics, 172, 2033-2045.

Greenhalgh, D., Hay, G., 1997. Mathematical modeling of the spread of HIV/AIDS amongst injecting drug users. IMA Journal of Mathematics Applied in Medicine \& Biology, 14, 1138.

Gyarmanthy, V.A. and Neiagus, A. (2006). The Effect of Personal Network Exposure on Injecting Equipment Sharing among IDUs in Budapest, Hungary. Connections, 27, 25-38. 
Hall, H.I, Song, R., Rhodes, P., Prejean, J., An, Q., Lee, L.M., Karon, J., Brookmeyer, R., Kaplan, E.H., McKenna, M.T., Janssen, R.S., for the HIV Incidence Surveillance Group, 2008. Estimation of HIV Incidence in the United States. Journal of the American Medical Association, 300, 520-529.

Hedström, P., 2006. Dissecting the Social. Cambridge: Cambridge University Press.

Hoffer, L.D., Bobashev G. and Morris, R.J. 2009. Researching a local heroin market as a complex adaptive system. American Journal of Community Psychology, 44, pp. 273-286.

Iannelli, M.F., Milner, A., Pugliese, A., Gonzo, M. 1997. The HIV/AIDS Epidemics among Drug Injectors: A Study of Contact Structure through a Mathematical Model. Mathematical Biosciences, 139, 25-58.

Janssen, M. A., Ostrom, E., 2006. Empirically Based, Agent-based models. Ecology and Society, 11(2), 37.

Kaplan, E.H., 1989. Needles that kill: Modeling human immunodeficiency virus transmission via shared drug injection equipment in shooting galleries. Reviews of Infectious Diseases, 11, 289-298.

Kiss, I.Z., Green, D.M., Kao, R.R., 2008. The effect of network mixing patterns on epidemic dynamics and the efficacy of disease contact tracing. Journal of the Royal Society Interface 5, 791-799.

Klein, H., Levy, J.A., 2003. Shooting gallery users and HIV risk. Journal of Drug Issues, 33, $751-767$.

Klovdahl, A.S., 1985. Social networks and the spread of infectious diseases: The AIDS example. Social Science \& Medicine, 21, 1203-1216. 
Klovdah., A.S., Graviss, E.A., Yaganehdoost, A., Ross, M.W., Wanger, A., Adams, G.J., Musser, J.M., 2001. Networks and tuberculosis: An undetected community outbreak involving public places. Social Science \& Medicine, 52, 681-694.

Kottiri, B.J., Friedman, S.R., Neaigus, A., Curtis, R., Des Jarlais, D.C., 2002. Risk networks and racial/ethnic differences in the prevalence of HIV infection among injection drug users. Journal of Acquired Immune Deficiency Syndromes, 30, 95-104.

Kretzschmar, M., Wiessing, L.G., 1998. Modeling the spread of HIV in social networks of injecting drug users. AIDS, 12, 801-811.

Latkin, C.A., Kuramoto, S.J., Davey-Rothwell, M.A., Tobin, K.E., 2010. Social Norms, Social Networks, and HIV Risk Behavior Among Injection Drug Users. AIDS Behavior, 14, $1159-1168$.

Latkin, C. Mandell, W., Vlahov. D., Knowlton, A., Oziemkowska, M., Celentano, D., 1995. Personal network characteristics as antecedents to needle-sharing and shooting gallery attendance. Social Networks, 17, 219-228.

Laufer, F.N., 2001. Cost-effectiveness of syringe exchange as an HIV prevention strategy. Journal of Acquired Immune Deficiency Syndromes, 28, 273-278.

Longini Jr., I.M., Clark, W.S., Haber, M., Horsburgh Jr, R., 1990. The stages of HIV infection: waiting times and infection transmission probabilities. In: Castillo-Chaves, C. (Ed.), Mathematical and Statistical Approaches to AIDS Epidemiology. New York: SpringerVerlag, pp. 111-137.

Macy, M.R., Willer, R., 2002. From factors to actors: Computational sociology and agent-based modeling. Annual Review of Sociology, 28,143-166. 
Morris, M., 1995. Data driven network models for the spread of infectious disease. In: Mollison, D. (Ed.), Epidemic Models: Their Structure and Relation to Data. Cambridge: Cambridge University Press, pp. 302-322.

Morris, M., Kretzschmar, M., 1997. Concurrent partnerships and the spread of HIV, AIDS, 11, 641-648.

Morris, M., Podhisita, C., Wawer, M.J., Handcock, M.S., 1996. Bridge populations in the spread of HIV/AIDS in Thailand. AIDS, 10, 1265-1271.

Moss, S., 2008. Alternative Approaches to the Empirical Validation of Agent-Based Models. Journal of Artificial Societies and Social Simulation, 11, 5.

Neaigus, A., Friedman, S.R., Curtis, R., Des Jarlais, D.C., Furst, R.T., Jose, B., Mota, P., Stepherson, B., Sufian, M., Ward, T., Wright, J.W., 1994. The relevance of drug injectors' social and risk networks for understanding and preventing HIV infection. Social Science \& Medicine, 38, 67-78.

Neaigus, A., Friedman, S.R., Goldstein, M.R., Ildefonso, G., Curtis, R., Jose, B., 1995. Using dyadic data for a network analysis of HIV infection and risk behaviors among injecting drug users. In: R.H. Needle, Coyle, S.L., Genser, S.G., Trotter, R.T. (Eds.), Social Networks, Drug Abuse, and HIV Transmission. NIDA Research Monograph 151, pp.20-37. Ouellet, L., Huo, D., Bailey, S.L., 2004. HIV risk practices among needle exchange users and nonusers in Chicago. Journal of Acquired Immune Deficiency Syndromes, 37, 1187-1196.

Peterson, D., Willard, K., Altmann, M., Gatewood, L., Davidson, G., 1990. Monte carlo simulation of HIV infection in an intravenous drug user community. Journal of Acquired Immune Deficiency Syndromes, 3, 1086-1095. 
Potterat J.J., Rothenberg, R.B., Muth, S.Q., 1999. Network structural dynamics and infectious disease propagation. International Journal of STD \& AIDS, 10, 182-185.

Pouget, E.R., Deren, S., Fuller, C.M., Blaney, S., McMahon, J.M., Kang, S.Y., Tortu, S., Andia, J.F., Des Jarlais, D.C., Vlahov, D., 2005. Receptive syringe sharing among injection drug users in Harlem and the Bronx during the New York State Expanded Syringe Access Demonstration Program. Journal of Acquired Immune Deficiency Syndromes, 39, 471-477.

Poteete. A., Janssen, M. A., Ostrom, E., 2010. Working together: collective action, the commons, and multiple methods in practice. Princeton, NJ: Princeton University Press.

Price, R.K., Cottler, L.B., Mager, D., Murray, K.S., 1995. Injecting drug use, characteristics of significant others, and HIV-risk behaviors. In: R.H. Needle, Coyle, S.L., Genser, S.G., Trotter, R.T. (Eds.), Social Networks, Drug Abuse, and HIV Transmission. NIDA Research Monograph 151, pp.38-59.

Raboud, J.M., Boily, M.C., Rajeswaran, J., O’Shaughnessy, M.V., Schechter, M.T., 2003. The impact of needle-exchange programs on the spread of HIV among injection drug users: a simulation study. Journal of Urban Health, 80, 302-20.

Rahmandad, H. and Sterman, J. 2008. Heterogeneity and Network Structure in the Dynamics of Diffusion: Comparing Agent-Based and Differential Equation Models. Management Science, 54, 998-1014.

Rhodes, T., Singer, M., Bourgois, P., Friedman, S.R., Strathdee, S.A., 2005. The social structural production of HIV risk among injecting drug users. Social Science \& Medicine, 61, 10261044.

Rogers, E. M. 2003. Diffusion of innovations. 5th ed. New York, NY: Free Press. 
Rothenberg, R.B., Baldwin, J., Trotter, R., Muth, S. 2001. The risk environment for HIV transmission: Results from the Atlanta and Flagstaff network studies. Journal of Urban Health, 78, 419-432.

Rothenberg R.B., Potterat, J.J., Wookhouse, D.E., Muth, S.Q., Darrow, W.W., Klovdahl, A.S., 1995. Social networks in disease transmission: the Colorado Springs study. In: R.H. Needle, Coyle, S.L., Genser, S.G., Trotter, R.T. (Eds.), Social Networks, Drug Abuse, and HIV Transmission. NIDA Research Monograph 151, pp.3-19.

Sallach, D.L., Macal. C.M., 2001. Introduction: The simulation of social agents. Social Science Computer Review, 19, 245-248.

Sherman, S.G., Latkin, C.A., Gielen, A.C., 2001. Social factors related to syringe sharing among injecting partners: A focus on gender. Substance Use \& Misuse, 36, 2113-2136.

Tyler, K., 2008. Social network characteristics and risky sexual and drug related behaviors among homeless young adults. Social Science Research, 37, 673-685.

UNAIDS, 2010. Report on the Global AIDS Epidemic. Geneva: UNAIDS.

Valente, T.W., 2005. Network models and methods for studying the diffusion of innovations. In: P. J. Carrington, Scott, J. and Wasserman, S. (Eds), Models and methods in social network analysis. New York, NY: Cambridge University Press.

Volz, E.M., Miller, J.C., Galvani, A., Ancel Meyers, L., 2011. Effects of Heterogeneous and Clustered Contact Patterns on Infectious Disease Dynamics. PLoS Computational Biolology 7, e1002042.

Volz, E.M., Frost, S.D., Rothenberg, R., Meyers, L.A., 2010. Epidemiological bridging by injection drug use drives an early HIV epidemic. Epidemics, 2, 155-164. 
Watts, C.H., May, R.M., 1992. The influence of concurrent partnerships on the dynamics of HIV/AIDS. Mathematical Bioscience, 108, 89-104.

Wilensky, U., 1999. NetLogo. Center for Connected Learning and Computer-Based Modeling, Northwestern University, Evanston, IL. http://ccl.northwestern.edu/netlogo/.

Windrum, P., Fagiolo, G., Moneta, A., 2007. Empirical Validation of Agent-Based Models: Alternatives and Prospects. Journal of Artificial Societies and Social Simulation, 10, 8. Wood, E., Tyndall, M.W., Spittal, P.M., Li, K., Kerr, T., Hogg, R.S., Montaner, J.S., O'Shaughnessy, M.V., Schechter, M.T., 2001. Unsafe injection practices in a cohort of injection drug users in Vancouver: Could safer injecting rooms help? Canadian Medical Association Journal, 165, 405-410.

Zaric, G., 2002 Random vs. nonrandom missing in network epidemic models, Health Care Management Science, 5, 147-155. 
Table 1. Data used for model calibration across core, inner periphery and outer periphery subgroups

\begin{tabular}{lcccc}
\hline \multicolumn{1}{c}{ Variable } & Core & Inner & Outer & Source $^{\mathrm{a}}$ \\
\hline Number of agents & 40 & 95 & 632 & $\mathrm{C}, \mathrm{N}$ \\
Average number of injecting partners & 12.8 & 5.2 & 0.5 & $\mathrm{C}$ \\
Injections (mean per person per month) & 395 & 193 & 60 & $\mathrm{C}$ \\
Agents who share used syringes & 15 & 33 & 115 & $\mathrm{C}$ \\
$\begin{array}{l}\text { Probability that syringe is shared } \\
\text { Agents who attend shooting galleries }\end{array}$ & 0.5 & 0.4 & 0.3 & $\mathrm{~L}, \mathrm{O}$ \\
$\begin{array}{l}\text { Probability that injection occurs in a } \\
\text { shooting gallery }^{\text {b }}\end{array}$ & 0.5 & 34 & 92 & $\mathrm{C}$ \\
\hline
\end{tabular}

Note: ${ }^{a} C=$ Curtis et al. (1995), N=Nieagus et al. (1995), L=Laufer (2001), O = Ouellet, Huo, and Bailey (2004); ${ }^{b}$ sources used to infer plausible values

Table 2. Derived preferences for network ties between core, inner periphery and outer periphery subgroups

\begin{tabular}{llccc}
\hline & & \multicolumn{3}{c}{ Preferences for Alter } \\
& & Core & Inner & Outer \\
\hline \multirow{3}{*}{ Ego } & Core & 0.6 & 0.3 & 0.1 \\
& Inner & 0.6 & 0.3 & 0.1 \\
& Outer & 0.6 & 0.2 & 0.2 \\
\hline
\end{tabular}

Note: Based on qualitative descriptions in Curtis et al. (1995)

Table 3. Overall network totals derived for model calibration

Variable Derived Totals

Network size (all agents)

Agents who share used syringes

Agents attending shooting galleries

Number of non-directed ties

Injections per month

Injections with shared syringes

Injections in shooting galleries 
Table 4. Parameters describing the overall and mean monthly injection behavior of core, inner periphery ${ }^{\mathrm{a}}$ and outer periphery network members across four agent based models

\begin{tabular}{|c|c|c|c|c|c|c|c|c|c|c|}
\hline & \multirow[t]{2}{*}{ Model $1^{\mathrm{a}}$} & \multicolumn{3}{|c|}{ Model $2^{\mathrm{b}}$} & \multicolumn{3}{|c|}{ Model $3^{c}$} & \multicolumn{3}{|c|}{ Model $4^{\mathrm{d}}$} \\
\hline & & $\mathrm{C}$ & I & $\mathrm{O}$ & $\mathrm{C}$ & I & $\mathrm{O}$ & $\mathrm{C}$ & I & $\mathrm{O}$ \\
\hline Agents (n) & 767 & 40 & 95 & 632 & 40 & 95 & 632 & 40 & 95 & 632 \\
\hline Average links (non-directed) & 0.86 & 12.8 & 5.2 & 0.5 & 12.8 & 5.2 & 0.5 & 12.8 & 5.2 & 0.5 \\
\hline HIV+ seeds & 6 & 2 & 2 & 2 & 2 & 2 & 2 & 2 & 2 & 2 \\
\hline Injections $^{\mathrm{f}}$ (per person) & 93.94 & & 93.94 & & 395 & 193 & 60 & 395 & 193 & 60 \\
\hline Syringe sharers & 163 & & 163 & & 15 & 33 & 115 & 15 & 33 & 115 \\
\hline Injections with shared syringes & 0.49 & & 0.49 & & 0.5 & 0.4 & 0.3 & 0.5 & 0.4 & 0.3 \\
\hline Shooting gallery users & & & & & & & & 20 & 34 & 92 \\
\hline Injections in shooting galleries & & & & & & & & 0.5 & 0.4 & 0.3 \\
\hline
\end{tabular}

Notes: $C=$ Core, $I=$ Inner periphery, $O=$ Outer periphery; ${ }^{a}$ Random network, random agent attributes; ${ }^{b}$ Calibrated network, random agent attributes; ${ }^{C}$ Calibrated network, calibrated agent attributes; ${ }^{d}$ Calibrated network, calibrated agent attributes, shooting gallery attendance; 
Table 5. Average number of HIV seropositive outcomes for each model by number of HIV infections and by proportion of network subgroup infected with HIV after 48 months

\begin{tabular}{|c|c|c|c|c|c|c|c|c|}
\hline & \multicolumn{2}{|c|}{ Model $1^{\mathrm{a}}$} & \multicolumn{2}{|c|}{ Model $2^{b}$} & \multicolumn{2}{|c|}{ Model $3^{c}$} & \multicolumn{2}{|c|}{ Model $4^{\mathrm{d}}$} \\
\hline & No. & Prop. & No. & Prop & No. & Prop & No. & Prop. \\
\hline $\begin{array}{l}\text { Total } \\
(n=767)\end{array}$ & 9.40 & 0.01 & 25.55 & 0.03 & 60.00 & 0.08 & 75.54 & 0.10 \\
\hline $\begin{array}{l}\text { Core } \\
(n=40)\end{array}$ & - & - & 6.74 & 0.17 & 15.47 & 0.39 & 16.00 & 0.40 \\
\hline $\begin{array}{l}\text { Inner Periphery } \\
(n=95)\end{array}$ & - & - & 10.51 & 0.11 & 28.70 & 0.30 & 31.40 & 0.33 \\
\hline $\begin{array}{l}\text { Outer Periphery } \\
(\mathrm{n}=632)\end{array}$ & - & - & 8.30 & 0.01 & 15.80 & 0.03 & 28.15 & 0.05 \\
\hline
\end{tabular}




\section{FIGURE CAPTIONS AND PREVIEWS}

Figure 1. Histograms showing distributions of the number of HIV infections produced across model runs (1000 runs/model) after 48 months

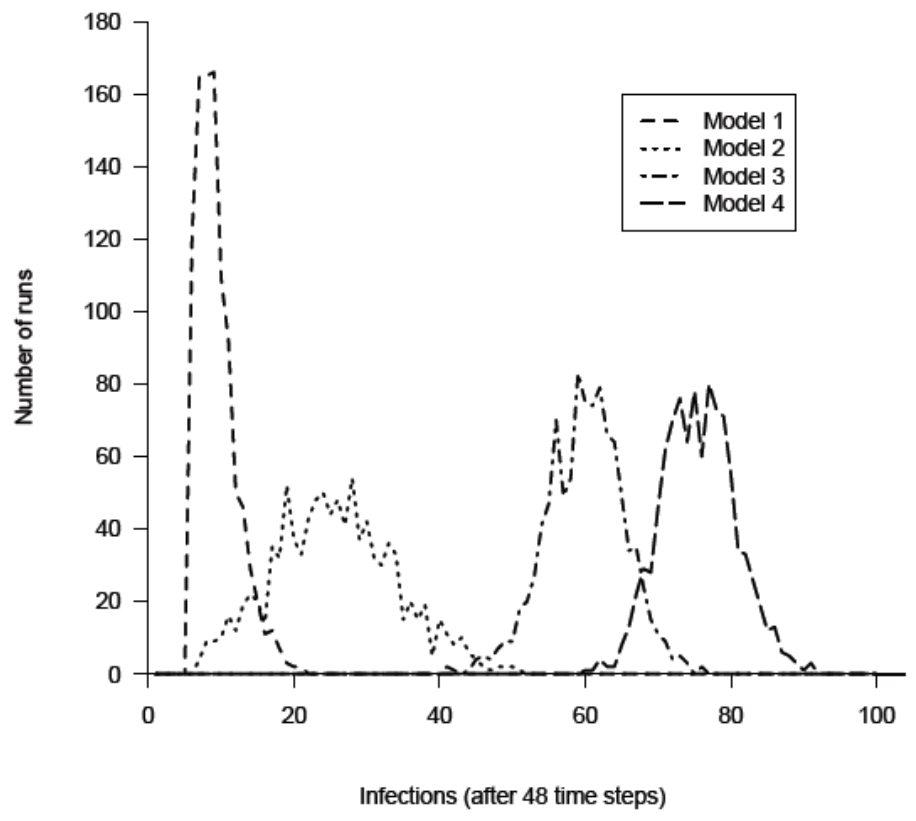


Figure 2A. Disease diffusion curves showing levels of HIV infection over time, by number of HIV infections and by proportion of network subgroup infected - Model 1
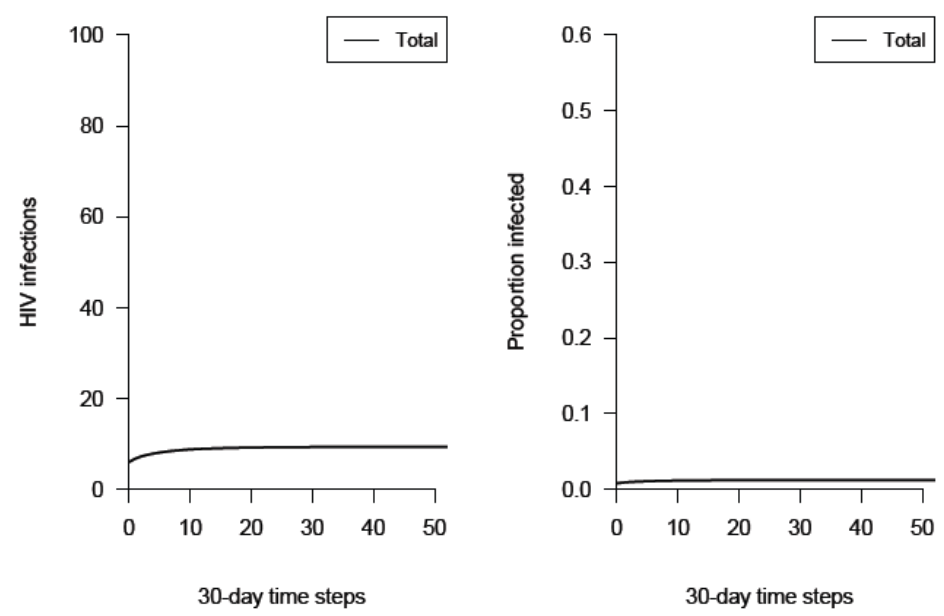

Figure 2B. Disease diffusion curves showing levels of HIV infection over time, by number of HIV infections and by proportion of network subgroup infected - Model 2
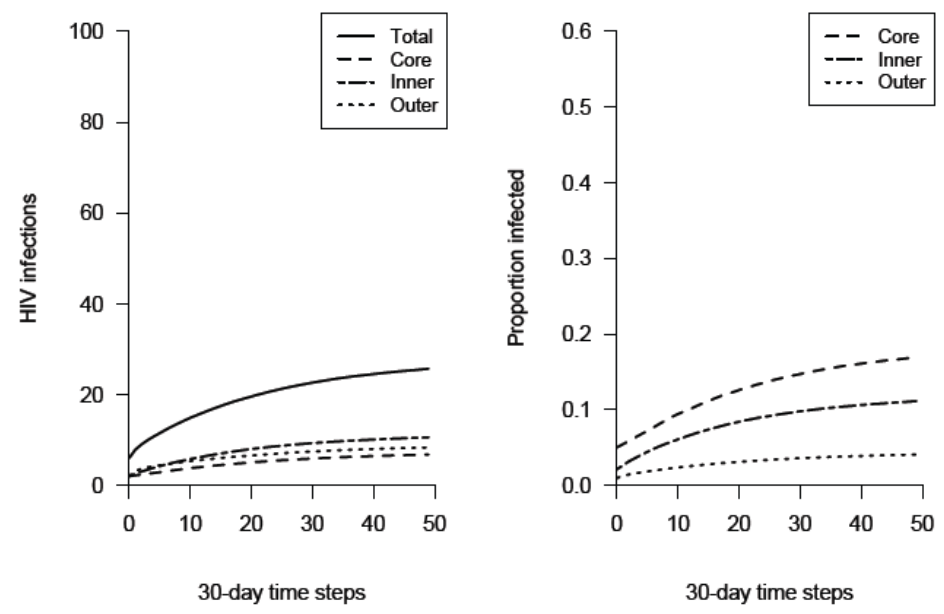
Figure 2C. Disease diffusion curves showing levels of HIV infection over time, by number of HIV infections and by proportion of network subgroup infected - Model 3
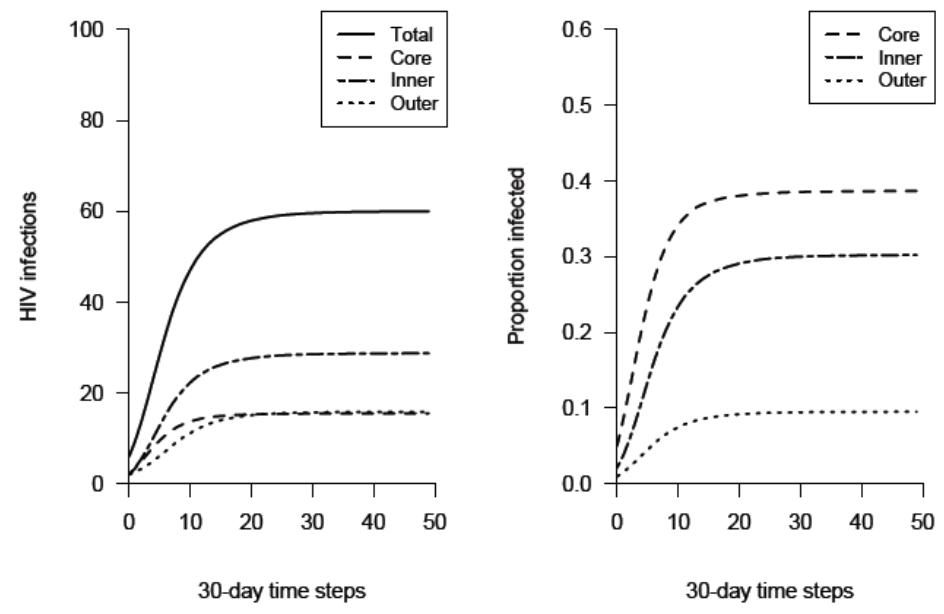

Figure 2D. Disease diffusion curves showing levels of HIV infection over time, by number of HIV infections and by proportion of network subgroup infected - Model 4
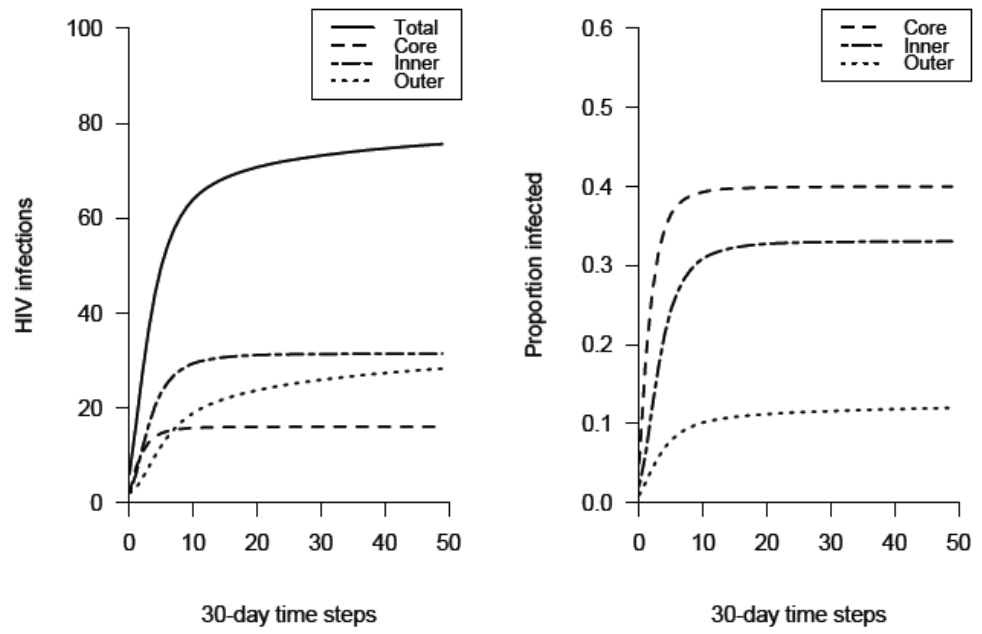